\title{
Common and unique mechanisms regulate fibrosis in various fibroproliferative diseases
}

\author{
Thomas A. Wynn
}

Immunopathogenesis Section, National Institute of Allergy and Infectious Diseases, NIH, Bethesda, Maryland, USA.

\begin{abstract}
Fibroproliferative diseases, including the pulmonary fibroses, systemic sclerosis, liver cirrhosis, cardiovascular disease, progressive kidney disease, and macular degeneration, are a leading cause of morbidity and mortality and can affect all tissues and organ systems. Fibrotic tissue remodeling can also influence cancer metastasis and accelerate chronic graft rejection in transplant recipients. Nevertheless, despite its enormous impact on human health, there are currently no approved treatments that directly target the mechanism(s) of fibrosis. The primary goals of this Review series on fibrotic diseases are to discuss some of the major fibroproliferative diseases and to identify the common and unique mechanisms of fibrogenesis that might be exploited in the development of effective antifibrotic therapies.
\end{abstract}

\section{Introduction}

Fibrosis is often defined as a wound-healing response that has gone out of control. Repair of damaged tissues is a fundamental biological process that allows the ordered replacement of dead or damaged cells after injury, a mechanism that is critically important for survival. Damage to tissues can result from various acute or chronic stimuli, including infections, autoimmune reactions, and mechanical injury. The repair process typically involves two distinct stages: a regenerative phase, where injured cells are replaced by cells of the same type, leaving no lasting evidence of damage; and a phase known as fibroplasia, or fibrosis, where connective tissue replaces normal parenchymal tissue. Although initially beneficial, the healing process becomes pathogenic if it continues unchecked, resulting in substantial remodeling of the ECM and formation of permanent scar tissue (Figure 1). In some cases, it might ultimately lead to organ failure and death.

In contrast to acute inflammatory reactions, which are characterized by rapidly resolving vascular changes, edema, and neutrophilic infiltration, pathogenic fibrosis typically results from chronic inflammatory reactions - defined as responses that persist for several weeks or months and in which inflammation, tissue destruction, and repair processes occur simultaneously. Despite having obvious etiological and clinical distinctions, most chronic fibrotic disorders have in common a persistent irritant that sustains the production of growth factors, proteolytic enzymes, angiogenic factors, and fibrogenic cytokines, which together stimulate the deposition of connective tissue elements that progressively remodel and destroy normal tissue architecture $(1,2)$.

When injuries occur, damaged epithelial and/or endothelial cells release inflammatory mediators that initiate an antifibrinolyticcoagulation cascade (3), which triggers formation of both blood clots and a provisional ECM (Figure 1). Platelets are exposed to ECM components, triggering aggregation, clot formation, and

Nonstandard abbreviations used: ARMD, age-related macular degeneration; EMT, epithelial-mesenchymal transition; HSC, hepatic stellate cell; IPF, idiopathic pulmonary fibrosis; PAMP, pathogen-associated molecular pattern; PRR, pattern recognition receptor; SSc, systemic sclerosis; TIMP, tissue inhibitor of metalloproteinases.

Conflict of interest: Thomas A. Wynn is employed by the NIH, US Department of Health and Human Services, which holds a patent position on the treatment of fibrosis by antagonizing IL-13 and the IL-13 receptor chains.

Citation for this article: J. Clin. Invest. 117:524-529 (2007). doi:10.1172/JCI31487. hemostasis. Next, platelet degranulation promotes vasodilation and increased blood vessel permeability, while stimulated myofibroblasts (collagen-secreting $\alpha$-SMA ${ }^{+}$fibroblasts) and epithelial and/or endothelial cells produce MMPs, which disrupt the basement membrane, allowing the efficient recruitment of inflammatory cells to the site of injury. Epithelial and endothelial cells also secrete growth factors, cytokines, and chemokines, which stimulate the proliferation and recruitment of leukocytes across the provisional ECM. Neutrophils are the most abundant inflammatory cell at the early stages of wound healing. When they degranulate and die, macrophages are recruited. During this initial leukocyte migration phase, the activated macrophages and neutrophils eliminate tissue debris, dead cells, and any invading organisms. They also produce cytokines and chemokines, which amplify the wound-healing response. These factors are also mitogenic and chemotactic for endothelial cells, which surround the injury and form new blood vessels as they migrate toward its center. Subsequently, T cells become activated and secrete profibrotic cytokines such as IL-13 and TGF- $\beta(4,5)$, which in turn further activate the macrophages and fibroblasts. Activated fibroblasts transform into $\alpha$-SMA-expressing myofibroblasts as they migrate along the fibrin lattice into the wound. Myofibroblasts are derived from local mesenchymal cells or recruited from the bone marrow (where they are known as fibrocytes) (Figure 1). Epithelial cells can also undergo epithelial-mesenchymal transition (EMT), providing a rich renewable source of myofibroblasts (6). Following activation, myofibroblasts promote wound contraction, the process in which the edges of the wound migrate toward the center. Finally, epithelial and/or endothelial cells divide and migrate over the basal layers to regenerate the damaged tissue, which completes the normal healing process. However, when repeated injury occurs, chronic inflammation and repair cause an excessive accumulation of ECM components (such as hyaluronic acid, fibronectin, proteoglycans, and interstitial collagens), which contribute to the formation of a permanent fibrotic scar.

The net amount of collagen deposited by fibroblasts is regulated continuously by collagen synthesis and collagen catabolism. The turnover of collagen and other ECM proteins is controlled by various MMPs and their inhibitors (tissue inhibitors of metalloproteinases [TIMPs]), which are produced by granulocytes, macrophages, epidermal cells, and myofibroblasts. Shifts in 


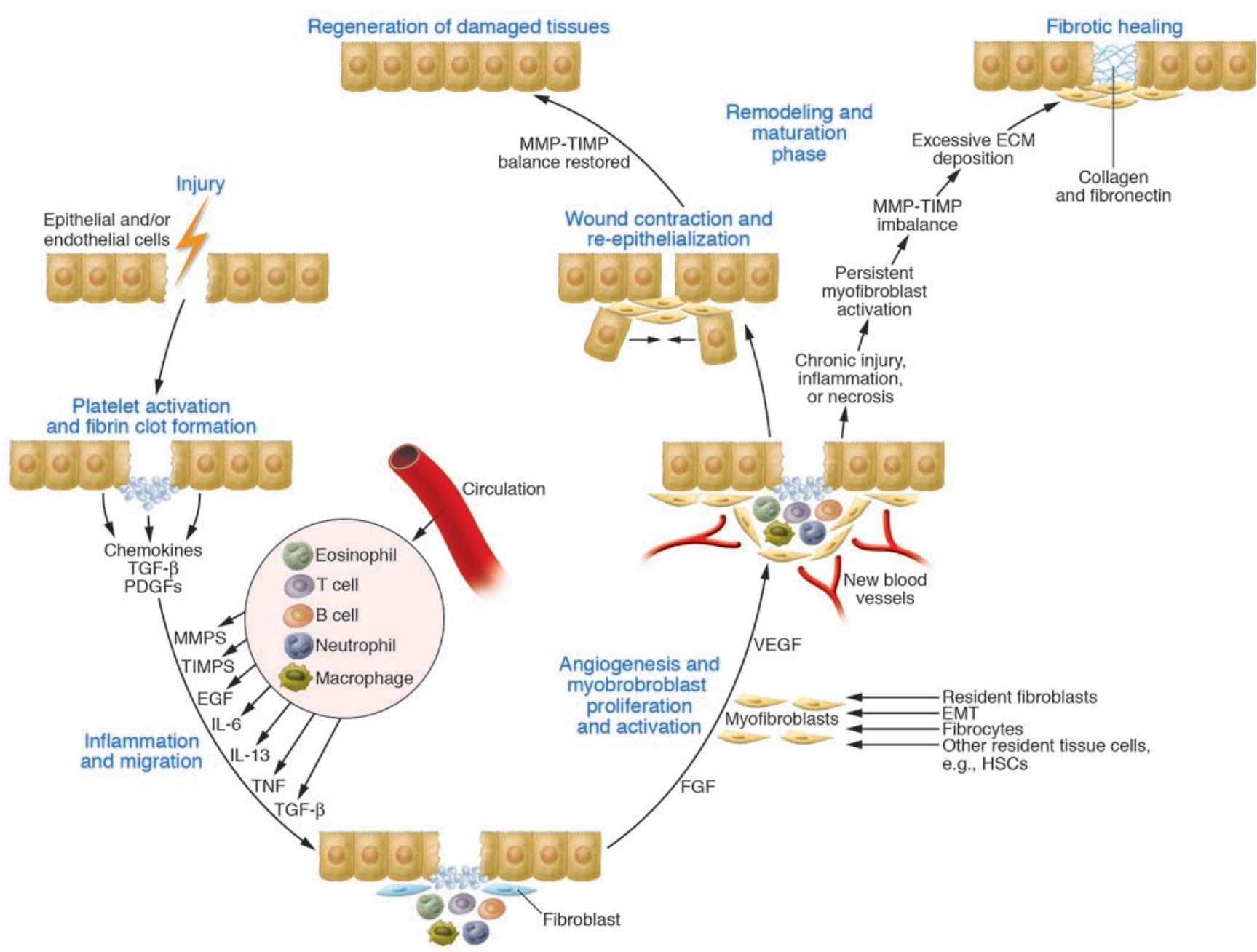

\section{Figure 1}

Outcomes of wound healing: tissue regeneration or fibrosis. Following tissue injury, epithelial and/or endothelial cells release inflammatory mediators that initiate an antifibrinolytic-coagulation cascade, which triggers blood clot formation. This is followed by an inflammatory and proliferative phase, when leukocytes are recruited and then activated and induced to proliferate by chemokines and growth factors. The activated leukocytes secrete profibrotic cytokines such as IL-13 and TGF- $\beta$. Stimulated epithelial cells, endothelial cells, and myofibroblasts also produce MMPs, which disrupt the basement membrane, and additional cytokines and chemokines that recruit and activate neutrophils, macrophages, T cells, B cells, and eosinophils, important components of reparative tissue. The activated macrophages and neutrophils clean up tissue debris, dead cells, and invading organisms. Shortly after the initial inflammatory phase, myofibroblasts produce ECM components, and endothelial cells form new blood vessels. The myofibroblasts can be derived from local mesenchymal cells, recruited from the bone marrow (where they are known as fibrocytes), or derived by EMT. In the subsequent remodeling and maturation phase, the activated myofibroblasts stimulate wound contraction. Collagen fibers also become more organized, blood vessels are restored to normal, scar tissue is eliminated, and epithelial and/or endothelial cells divide and migrate over the basal layers to regenerate the epithelium or endothelium, respectively, restoring the damaged tissue to its normal appearance. However, in the case of chronic wounds, the normal healing process is disrupted. Persistent inflammation, tissue necrosis, and infection lead to chronic myofibroblast activation and excessive accumulation of ECM components, which promotes the formation of a permanent fibrotic scar.

these opposing mechanisms (synthesis versus catabolism) regulate the net increase or decrease of collagen within a wound (7). The expanding pool of mesenchymal cells further exacerbates the response. In the remodeling phase, the synthesis of new collagen by fibroblasts exceeds the rate at which it is degraded such that the total amount of collagen continues to increase. Although inflammation typically precedes fibrosis, results from experimental models of this process have demonstrated that fibrosis is not necessarily driven by inflammation at all times, suggesting that the mechanisms that regulate fibrogenesis are, to a certain extent, distinct from those regulating inflammation (8). This might explain the general lack of efficacy of antiinflammatory mediators in the treatment of fibrotic disease and the need to identify targeted antifibrotic therapies.

The spectrum of diseases that result from chronic tissue damage or out-of-control wound-healing responses are too numerous to list. However, the goal of this Review series on fibrotic diseases is to highlight some of the major fibrotic diseases and to identify common and unique mechanisms of fibrogenesis in the various organ systems affected by these diseases. 


\section{The role of chronic infections and innate immunity}

The Review series begins with an article by Alessia Meneghin and Cory Hogaboam on the role of the innate immune system in the regulation of fibrosis induced following infection (9). They suggest that many fibrotic disorders have an infectious etiology, with bacteria, viruses, fungi, and multicellular parasites driving chronic inflammation and the development of fibrosis. Persistent infection with these organisms triggers marked alterations in the activation status of myofibroblasts and M2 macrophages (also known as alternatively activated macrophages), key cells involved in the remodeling process (8). They argue that constant exposure of fibroblasts and other structural cells to conserved pathogen-associated molecular patterns (PAMPs) maintains the cells at a heightened state of activation. PAMPs are pathogen byproducts such as lipoproteins, bacterial DNA, and double-stranded RNA, which are recognized by pattern recognition receptors (PRRs) expressed on a wide variety of cells (10). The interaction between PAMPs and PRRs serves as a first line of defense during infection and activates numerous proinflammatory cytokine and chemokine pathways. Therefore, when infections become chronic, persistent production of PAMPs provides a constant driving force to activate the innate immune system. Because fibroblasts express PRRs (such as TLRs), Meneghin and Hogaboam propose the intriguing theory that TLR ligands are directly stimulating the differentiation of fibroblasts into activated myofibroblasts. Th2 cytokines can also augment the response by upregulating TLR9 expression on fibroblasts, which secrete the profibrotic chemokine CC chemokine ligand 2 (CCL2) when stimulated with TLR9 ligands (11). Therefore, they suggest that inhibiting pathogen-mediated fibroblast activation might represent a viable approach for antifibrotic therapy.

\section{Myofibroblasts}

Origins of the myofibroblast. The origins of the myofibroblast are discussed in many of the articles in this Review series. Although it was originally thought that activated local tissue fibroblasts were the primary producers of ECM components following injury (3), it is now widely believed that myofibroblasts are derived from at least two additional sources (6). In addition to resident mesenchymal fibroblasts, myofibroblasts can be derived from epithelial cells in a process known as EMT (12), and Bucala and colleagues have identified a unique circulating fibroblast-like cell that is derived from bone marrow stem cells (13). These blood-borne mesenchymal progenitors have a fibroblast- and/or myofibroblast-like phenotype (that is, they express CD34, CD45, and type I collagen) and were termed fibrocytes. Furthermore, in some tissues, resident fibroblasts are not the only source of myofibroblasts. For example, as discussed in the Review by John Iredale, it is thought that in liver fibrosis resident hepatic stellate cells (HSCs) are one of the main sources of myofibroblasts (14).

Because fibrocytes can extravasate into tissues and participate with resident mesenchymal cells in the reparative process, there has been growing interest in dissecting the role of fibrocytes in various fibroproliferative diseases (15). In their Review, Robert Strieter and colleagues provide a comprehensive overview of the role of fibrocytes in experimental models of pulmonary fibrosis and discuss their potential contribution to the development of idiopathic pulmonary fibrosis (IPF) in humans (16). Much of their recent work has focused on the role of the CXC chemokine receptor family in promoting the trafficking of various leukocytes, including the circulating fibrocyte, to sites of injury. In mice, CXC chemokine receptor 4 (CXCR4), CC chemokine receptor 7 (CCR7), and CCR2 seem to mediate recruitment of fibrocytes to the injured lung $(15,17)$. Because fibrocytes produce various factors that are involved in mediating fibroproliferation (6), interrupting critical chemokine signaling pathways could impact the pathogenesis of pulmonary fibroproliferative disorders by reducing fibrocyte recruitment. As such, Strieter and colleagues argue that a more detailed understanding of the mechanisms regulating fibrocyte differentiation, recruitment, and activation is needed. We also need to better understand the individual roles of fibrocytes, myofibroblasts derived by EMT, and resident mesenchymal cells in the fibrotic process, as it seems likely that all three populations are involved. Therefore, targeting fibrocytes alone might not be sufficient for all types of fibroproliferative diseases.

Paracrine and autocrine mechanisms regulate myofibroblast activation. In their Review, John Varga and David Abraham discuss how fibroblasts explanted from lesional skin or the fibrotic lungs of patients with systemic sclerosis (SSc) have a constitutively activated myofibroblast-like phenotype (18). This SSc phenotype is characterized by enhanced ECM synthesis, constitutive secretion of cytokines and chemokines, and increased expression of cell surface receptors $(19,20)$. Because most of the characteristics of fibroblasts from patients with SSc are reproduced in normal fibroblasts following stimulation with TGF- $\beta$, it was suggested that the SSc fibroblast phenotype is due to autocrine TGF- $\beta$ signaling. However, TGF- $\beta$ / SMAD3-independent mechanisms have also been proposed (21, 22). Recent evidence suggests that epigenetic changes in SSc fibroblasts might also be contributing to their persistent activation (23). Although the etiology of SSc is unknown, viral infections have been implicated, with human CMV infections driving the production of autoantibodies and connective tissue growth factor (CTGF), both of which can promote fibroblast activation $(20,24)$. B cells might also be involved, either by producing autoantibodies or by secreting IL-6, a known fibroblast growth factor (25). Still other studies have argued that Th2 cytokines are involved (26). Therefore, paracrine signals derived from activated lymphocytes, as well as autocrine mechanisms in fibroblasts, might be cooperating to promote and maintain the SSc fibroblast phenotype. Because inappropriate fibroblast activation is the central pathogenic mechanism in many fibrotic disorders, including SSc $(8,20)$, Varga and Abraham emphasize that to identify novel therapeutic targets, we need to better understand the mechanisms that govern mesenchymal cell transformation into activated myofibroblasts (18).

\section{The role of ANG II and TGF- $\beta 1$}

As discussed in the Review by Bradford Berk and colleagues, a role for TGF- $\beta$ has also been observed in ECM remodeling in hypertensive heart disease (27). They discuss how sustained hypertension causes structural and functional alterations in the heart, which lead to progressive diastolic and systolic dysfunction. They argue that ANG II, produced locally by activated macrophages and myofibroblasts, upregulates the expression of TGF- $\beta 1$, which drives perivascular fibrosis and scarring in the heart.

Berk and colleagues also discuss how structural changes in the ECM affect cell signaling, particularly in myocytes, leading to left ventricular hypertrophy and dysfunction. Although all major components of the renin-angiotensin-aldosterone system can exhibit profibrotic activity, ANG II seems to be the dominant hormone responsible for cardiac fibrosis in hypertensive heart disease (28). ANG II exerts its effects directly by stimulating TGF- $\beta 1$ production 


\title{
Antifibrotic strategies discussed in this Review series
}

Various antiinflammatory/immunosuppressive/cytotoxic drugs (including colchicine, azathioprine, cyclophosphamide, prednisone, thalidomide, pentoxifylline, and theophylline)

TGF- $\beta$ signaling modifiers (including relaxin, SMAD7, HGF, and BMP7, as well as TGF- $\beta 1$, TGF $\beta$ RI, TGF $\beta$ RII, EGR-1, and CTGF inhibitors)

Cytokine and cytokine receptor antagonists (inhibitors of IL-1 $\beta$, IL-5, IL-6, IL-13, IL-21, IL-4R, IL-13R $\alpha 1$, GM-CSF, TNF- $\alpha$, oncostatin M, WISP-1, and PDGFs)

Cytokines and chemokines (IFN- $\gamma$, IFN- $\alpha / \beta$, IL-12, IL-10, HGF, CXCL10, and CXCL11)

Chemokine antagonists (inhibitors of CXCL1, CXCL2, CXCL12, CCL2, CCL3, CCL6, CCL17, and CCL18)

Chemokine receptor antagonists (inhibitors of CCR2, CCR3, CCR5, CCR7, CXCR2, and CXCR4)

TLR antagonists (inhibitors of TLR3, TLR4, and TLR9)

Angiogenesis antagonists (VEGF-specific antibodies and adenosine deaminase replacement therapy)

Antihypertensive drugs (beta blockers and inhibitors of ANG II, ACE, and aldosterone)

Vasoactive substances (ET-1 receptor antagonists and bosetan)

Inhibitors of the enzymes that synthesize and process collagen (inhibitors of prolyl hydroxylase)

B cell antagonists (rituximab)

Integrin/adhesion molecule antagonists (molecules that block $\alpha_{1} \beta_{1}$ and $\alpha_{v} \beta_{6}$ integrins, as well as inhibitors of integrin linked kinase, and antibodies specific for ICAM-1 and VCAM-1)

Proapoptotic drugs that target myofibroblasts

MMP inhibitors (inhibitors of MMP2, MMP9, and MMP12)

TIMP inhibitors (antibodies specific for TIMP-1)

Gene silencing strategies and gene therapy (shRNA for TGF- $\beta 1$ and TGF $\beta$ RII)

Stem/progenitor cell transplantation technologies

Various combinations of the above

\begin{abstract}
ACE, ANG I converting enzyme; BMP7, bone morphogenetic protein 7; CCL, CC chemokine ligand; CCR, CC chemokine receptor; CTGF, connective tissue growth factor; CXCL, CXC chemokine ligand; CXCR, CXC chemokine receptor; EGR-1, early growth response 1; ET-1, endothelin-1; HGF, hepatocyte growth factor; shRNA, short hairpin RNA; TGF $\beta$ RI, TGF- $\beta$ receptor type I; WISP-1, Wnt-1-induced secreted protein 1.
\end{abstract}

and by triggering fibroblast proliferation and differentiation into collagen-secreting myofibroblasts (29). In addition to its effects on TGF- $\beta 1$ secretion and activation, ANG II directly enhances TGF- $\beta 1$ signaling by increasing SMAD2 levels and augmenting nuclear translocation of phosphorylated SMAD3. TGF- $\beta 1$ can, in turn, further augment the production of interstitial collagens, fibronectin, and proteoglycans by cardiac myofibroblasts (1). TGF- $\beta 1$ also triggers its own production by myofibroblasts, thereby establishing an autocrine cycle of myofibroblast differentiation and activation. Overexpression of TGF- $\beta 1$ in transgenic mice results in cardiac hypertrophy characterized by both interstitial fibrosis and hypertrophic growth of cardiac myocytes (30). Patients suffering from idiopathic hypertrophic cardiomyopathy and dilated cardiomyopathy also have increased levels of TGF- $\beta 1$ in the left ventricular myocardium (31). Therefore, therapies that target the renin-angiotensin-aldosterone system or TGF- $\beta 1$ pathways might provide effective strategies to slow the progression of fibrosis in hypertensive heart disease.

\section{ECM remodeling and reversibility of intractable fibrosis}

In addition to outlining the contribution of HSCs to the myofibroblast population in liver fibrosis, Iredale discusses the emerging paradigm that fibrosis is a reversible process (14). Liver fibrosis and end-stage cirrhosis are the key pathological features of many chronic liver diseases $(32,33)$. Hepatic fibrosis can lead to portal hypertension and liver failure and is associated with an increased risk of liver cancer (34). Although liver fibrosis was at one time considered a progressive and irreversible disease, data from animal models and human studies have begun to challenge the dogma that fibrosis is irreversible (35). Successful elimination of HBV and HCV in chronically infected individuals is often associated with marked regression of fibrosis, providing evidence that human liver fibrosis is at least partially reversible (35). Similar observations have also been reported in patients with schistosomiasis following treatment with praziquantel, which eliminates the causative pathogen - a Schistosoma spp. (36). However, it remains controversial whether advanced fibrosis can be reversed to the level where normal liver architecture is restored. There is substantial experimental evidence that if cirrhosis is sufficiently advanced, reversal is no longer possible. In his Review, Iredale discusses in detail how liver fibrosis is regulated by dynamic interactions between MMPs and their inhibitors, the TIMPs. Fibrosis occurs when the balance between TIMPs and MMPs tips in favor of TIMPs, whereas resolution is associated with reduced TIMP expression. Interestingly, because advanced fibrosis is relatively hypocellular, it has been suggested that incomplete ECM degradation (that is, irreversible fibrosis) develops when the appropriate cellular mediators (the source of MMPs) are no longer present (37). This suggests that ongoing inflammation might be required for the successful resolution of fibrosis. Not surprisingly, the identity and source of key MMPs that mediate the resolution of fibrosis are being intensively investigated. Recent studies have demonstrated that macrophage depletion at the onset of fibrosis resolution can retard ECM degradation and the loss of activated HSCs (38). This suggests that macrophages might be essential for initiating ECM degradation, perhaps by producing specific MMPs. Therefore, it might be possible to identify specific mechanisms or pathways that can be exploited to reverse what was once thought to be intractable scarring. 


\section{A link between angiogenesis and fibrogenesis}

The Reviews by Strieter and colleagues, Varga and Abraham, and Martin Friedlander describe the important connections between angiogenesis, vascular remodeling, and fibrosis $(16,18,39)$. The balance between proangiogenic and antiangiogenic factors regulates vascular remodeling in various fibrotic diseases, including pulmonary fibrosis, SSc, and many fibrotic diseases of the eye (20, $40)$. In addition to fibroproliferation and deposition of ECM components, the pathogenesis of IPF, which ultimately leads to endstage lung fibrosis, is characterized by substantial vascular remodeling. Nevertheless, few studies have considered the important contribution of vascular remodeling to the progression of fibrosis in the lung. Strieter and colleagues discuss how distinct members of the CXC chemokine family can exhibit either angiogenic or angiostatic activity and relate this information to the progression of pulmonary fibrosis (16). They maintain that vascular remodeling is critically connected to the mechanisms of tissue fibrogenesis and that targeting the CXC chemokine family might offer a unique approach to treat fibrotic disease in general.

As discussed in the Review by Varga and Abraham, vascular injury and damage are also a prominent and early manifestation of SSc (18). Patients with SSc are commonly classified into two distinct subsets on the basis of the pattern of skin involvement. Diffuse cutaneous SSc is characterized by rapidly progressive fibrosis of the skin and lungs, whereas limited cutaneous SSc is dominated by vascular manifestations, with minimal fibrosis of the skin and internal organs. The loss of microvasculature in SSc is associated with substantial tissue hypoxia, which induces the expression of VEGF and its receptors (41). Nevertheless, despite developing a strong angiogenic environment, SSc is associated with impaired angiogenesis and progressive disappearance of blood vessels (20). Varga and Abraham discuss this apparent paradox and suggest that reduced numbers of circulating bone marrow-derived CD $34^{+}$ endothelial progenitor cells, as well as their impaired differentiation into mature endothelial cells, might be contributing to the vascular defects, as was recently demonstrated in a study by Del Papa et al. (42). Whether the reduction in circulating endothelial progenitor cells in SSc is due to their depletion in the bone marrow, destruction in the periphery, or some other mechanism, however, remains unresolved.

The final article in this series, by Friedlander, highlights the important role of vascular remodeling in the development of various fibrotic eye diseases (39). The end result of many eye diseases is fibrosis or scar formation, which can ultimately impair vision. For example, certain viral and parasitic infections can result in fibrosis and opacification of the cornea and uncontrolled vascular proliferation in the eye resulting from diabetes-associated retinal hypoxia can lead to fibrosis and traction retinal detachments, a serious complication of advanced diabetic retinopathy (43). Under the retina, similar fibrovascular scarring can occur subsequent to subretinal hemorrhage that is associated with neovascular agerelated macular degeneration (ARMD) (44). Collectively, these conditions of fibrosis in the eye lead to vision loss in millions of people worldwide. In his Review, Friedlander discusses the cellular pathophysiology associated with fibrosis in the anterior (cornea and trabecular meshwork) and posterior segments (retina and vitreous) of the eye. The final common pathway for many of these diseases is injury resulting in inflammatory changes, tissue edema, hypoxia-driven neovascularization, and ultimately fibrosis. Once new blood vessels begin to grow in the eye, they are prone to rup- ture, leading to further activation of wound-healing responses and ultimately the development of severe fibrosis. Therefore, prevention of the primary vascular abnormality has been the most promising therapeutic strategy for the treatment of these fibrotic diseases of the eye.

\section{Human embryonic stem cell-based therapies for fibrosis}

As discussed above, current approaches aimed at treating ARMD and other diseases of the eye are primarily directed at inhibiting promoters of angiogenesis, such as VEGF, cytokines, specific MMPs, and adhesion molecules (integrins) (44). Unfortunately, pharmacological interventions are typically initiated at an advanced stage of disease and do not target the underlying pathophysiology; therefore, they often fail to substantially impact disease progression. A more successful therapeutic approach would involve replacing damaged cells or restoring homeostasis to the areas that underlie the fibrotic response (45). In his Review, Friedlander describes how cell-based therapies using adult bone marrow-derived progenitor/ stem cell technologies might be used to treat currently incurable fibrovascular diseases of the eye, such as ARMD. Similar stem cell therapies have already proven successful at restoring cardiac function in injured hearts (46); therefore, they might ultimately prove successful for a wide variety of fibroproliferative diseases.

\section{The challenge ahead: clinical trial design and endpoints}

Although a great deal of work is still needed to fully understand the mechanisms of fibrogenesis, a substantial amount of progress has been made over the past few years. The work of my own laboratory has focused on the mechanisms regulating liver fibrosis following infection with the helminth parasite Schistosoma mansoni. In this work, the Th2 cytokine IL-13 was identified as a dominant mediator of fibrosis (47), and more recently, an important antifibrotic role for the IL-13 decoy receptor, IL-13R $\alpha 2$, was described (48). We have also shown that an IL-13 inhibitor can be used to completely halt the progression of fibrosis, despite ongoing infection (49). Interestingly, IL-13 antagonism had little impact on the inflammatory response, at least at the early stages of the disease $(47,49)$. Therefore, it seems that successful antifibrotic therapy does not necessarily depend on inhibiting inflammation or removing the etiologic agent. These data suggest that it might be possible to develop therapeutics that directly target the fibrotic machinery. As illustrated in many of the articles in this Review series, there is a growing list of novel mediators and pathways, in addition to IL-13 that could be developed as antifibrotic treatments. These include chemokine and TLR antagonists, angiogenesis inhibitors, antihypertensive drugs, TGF- $\beta$-signaling modifiers, B cell-depleting antibodies, and stem/progenitor cell transplantation technologies (see "Antifibrotic strategies discussed in this Review series"). As there are many potential targets and strategies, what we need now is a coherent plan for translating the available experimental information into clinical practice. However, there are many difficult challenges ahead that must be overcome before any treatment reaches the clinic. The most difficult obstacle will be to design effective clinical trials with well defined clinical endpoints. Because fibrosis typically progresses slowly in most diseases, clinical trials could be long and expensive. Therefore, there is a desperate need to develop noninvasive methods, such as serum markers or improved imaging techniques, to quickly quantify changes in the natural history of a disease. Nearly $45 \%$ of all deaths in the developed world are attributed to some type of chronic fibropro- 
liferative disease. Therefore, the demand for antifibrotic drugs that are both safe and effective is likely to be enormous, so it is hoped that researchers, funding agencies, and the pharmaceutical industry will continue to accelerate their efforts to identify and develop effective antifibrotic therapies.

1. Tomasek, J.J., Gabbiani, G., Hinz, B., Chaponnier, C., and Brown, R.A. 2002. Myofibroblasts and mechano-regulation of connective tissue remodelling. Nat. Rev. Mol. Cell Biol. 3:349-363.

2. Friedman, S.L. 2004. Mechanisms of disease: mechanisms of hepatic fibrosis and therapeutic implications. Nat. Clin. Pract. Gastroenterol. Hepatol. 1:98-105.

3. Kumar, V., Abbas, A.K., and Fausto, N. 2005. Tissue renewal and repair: regeneration, healing, and fibrosis. In Pathologic basis of disease. V. Kumar, A.K. Abbas, and N. Fausto, editors. Elsevier Saunders. Philadelphia, Pennsylvania, USA. 87-118.

4. Li, M.O., Wan, Y.Y., Sanjabi, S., Robertson, A.K., and Flavell, R.A. 2006. Transforming growth factor-beta regulation of immune responses. Annu. Rev. Immunol. 24:99-146.

5. Wynn, T.A. 2003. IL-13 effector functions. Annu. Rev. Immunol. 21:425-456.

6. Quan, T.E., Cowper, S.E., and Bucala, R. 2006. The role of circulating fibrocytes in fibrosis. Curr. Rheumatol. Rep. 8:145-150.

7. Pardo, A., and Selman, M. 2006. Matrix metalloproteases in aberrant fibrotic tissue remodeling. Proc. Am. Thorac. Soc. 3:383-388.

8. Wynn, T.A. 2004. Fibrotic disease and the T(H)1/ $\mathrm{T}(\mathrm{H}) 2$ paradigm. Nat. Rev. Immunol. 4:583-594.

9. Meneghin, A., and Hogaboam, C.M. 2007. Infectious disease, the innate immune response, and fibrosis. J. Clin. Invest. 117:530-538. doi:10.1172/JCI30595.

10. Akira, S., and Takeda, K. 2004. Toll-like receptor signalling. Nat. Rev. Immunol. 4:499-511.

11. Coelho, A.L., Hogaboam, C.M., and Kunkel, S.L. 2005. Chemokines provide the sustained inflammatory bridge between innate and acquired immunity. Cytokine Growth Factor Rev. 16:553-560.

12. Kalluri, R., and Neilson, E.G. 2003. Epithelialmesenchymal transition and its implications for fibrosis. J. Clin. Invest. 112:1776-1784. doi:10.1172/ JCI200320530.

13. Bucala, R., Spiegel, L.A., Chesney, J., Hogan, M., and Cerami, A. 1994. Circulating fibrocytes define a new leukocyte subpopulation that mediates tissue repair. Mol. Med. 1:71-81.

14. Iredale, J.P. 2007. Models of liver fibrosis: exploring the dynamic nature of inflammation and repair in a solid organ. J. Clin. Invest. 117:539-548. doi:10.1172/JCI30542.

15. Phillips, R.J., et al. 2004. Circulating fibrocytes traffic to the lungs in response to CXCL12 and mediate fibrosis. J. Clin. Invest. 114:438-446. doi:10.1172/ JCI200420997.

16. Strieter, R.M., Gomperts, B.N., and Keane, M.P. 2007. The role of CXC chemokines in pulmonary fibrosis. J. Clin. Invest. 117:549-556. doi:10.1172/ JCI30562.

Address correspondence to: Thomas A. Wynn, Immunopathogenesis Section, National Institute of Allergy and Infectious Diseases, NIH, 50 South Drive, Room 6154, MSC 8003, Bethesda, Maryland 20892, USA. Phone: (301) 496-4758; Fax: (301) 480-5025; E-mail: twynn@niaid.nih.gov.

17. Moore, B.B., et al. 2005. CCR2-mediated recruitment of fibrocytes to the alveolar space after fibrotic injury. Am. J. Pathol. 166:675-684.

18. Varga, J., and Abraham, D. 2007. Systemic sclerosis: a prototypic multisystem fibrotic disorder. J. Clin. Invest. 117:557-567. doi:10.1172/JCI31139.

19. Strehlow, D., and Korn, J.H. 1998. Biology of the scleroderma fibroblast. Curr. Opin. Rheumatol. 10:572-578.

20. Abraham, D.J., and Varga, J. 2005. Scleroderma: from cell and molecular mechanisms to disease models. Trends Immunol. 26:587-595.

21. Kaviratne, M., et al. 2004. IL-13 activates a mechanism of tissue fibrosis that is completely TGF-beta independent. J. Immunol. 173:4020-4029.

22. Moustakas, A., and Heldin, C.H. 2005. Non-Smad TGF-beta signals. J. Cell Sci. 118:3573-3584.

23. Wang, Y., Fan, P.S., and Kahaleh, B. 2006. Association between enhanced type I collagen expression and epigenetic repression of the FLI1 gene in scleroderma fibroblasts. Arthritis Rheum. 54:2271-2279.

24. Markiewicz, M., et al. 2004. The 72-kilodalton IE-1 protein of human cytomegalovirus (HCMV) is a potent inducer of connective tissue growth factor (CTGF) in human dermal fibroblasts. Clin. Exp. Rheumatol. 22(Suppl. 33):S31-S34.

25. Hasegawa, M., Fujimoto, M., Takehara, K., and Sato, S. 2005. Pathogenesis of systemic sclerosis: altered $\mathrm{B}$ cell function is the key linking systemic autoimmunity and tissue fibrosis. J. Dermatol. Sci. 39:1-7.

26. Ong, C.J., et al. 1999. A role for T helper 2 cells in mediating skin fibrosis in tight-skin mice. Cell Immunol. 196:60-68.

27. Berk, B.C., Fujiwara, K., and Lehoux, S. 2007. ECM remodeling in hypertensive heart disease. J. Clin. Invest. 117:568-575. doi:10.1172/JCI31044.

28. Watanabe, T., Barker, T.A., and Berk, B.C. 2005. Angiotensin II and the endothelium: diverse signals and effects. Hypertension. 45:163-169.

29. Rosenkranz, S. 2004. TGF-beta1 and angiotensin networking in cardiac remodeling. Cardiovasc. Res. 63:423-432.

30. Rosenkranz, S., et al. 2002. Alterations of betaadrenergic signaling and cardiac hypertrophy in transgenic mice overexpressing TGF-beta(1). Am. J. Physiol. Heart Circ. Physiol. 283:H1253-H1262.

31. Li, R.K., et al. 1997. Overexpression of transforming growth factor-beta1 and insulin-like growth factor-I in patients with idiopathic hypertrophic cardiomyopathy. Circulation. 96:874-881.

32. Albanis, E., and Friedman, S.L. 2006. Antifibrotic agents for liver disease. Am. J. Transplant. 6:12-19.

33. Bataller, R., and Brenner, D.A. 2005. Liver fibrosis. J. Clin. Invest. 115:209-218. doi:10.1172/ JCI200524282.

34. Llovet, J.M., et al. 2006. A molecular signature to discriminate dysplastic nodules from early hepatocellular carcinoma in HCV cirrhosis. Gastroenterology. 131:1758-1767.

35. Fallowfield, J.A., Kendall, T.J., and Iredale, J.P. 2006. Reversal of fibrosis: no longer a pipe dream? Clin. Liver Dis. 10:481-497.

36. Vennervald, B.J., and Dunne, D.W. 2004. Morbidity in schistosomiasis: an update. Curr. Opin. Infect. Dis. 17:439-447.

37. Issa, R., et al. 2004. Spontaneous recovery from micronodular cirrhosis: evidence for incomplete resolution associated with matrix cross-linking. Gastroenterology. 126:1795-1808.

38. Duffield, J.S., et al. 2005. Selective depletion of macrophages reveals distinct, opposing roles during liver injury and repair. J. Clin. Invest. 115:56-65. doi:10.1172/JCI200522675.

39. Friedlander, M. 2007. Fibrosis and diseases of the eye. J. Clin. Invest. 117:576-586. doi:10.1172/JCI31030.

40. Strieter, R.M., Belperio, J.A., and Keane, M.P. 2002. $\mathrm{CXC}$ chemokines in angiogenesis related to pulmonary fibrosis. Chest. 122(Suppl. 6):298S-301S.

41. Distler, O., et al. 2002. Angiogenic and angiostatic factors in systemic sclerosis: increased levels of vascular endothelial growth factor are a feature of the earliest disease stages and are associated with the absence of fingertip ulcers. Arthritis Res. 4:R11.

42. Del Papa, N., et al. 2006. Bone marrow endothelial progenitors are defective in systemic sclerosis. Arthritis Rheum. 54:2605-2615.

43. Wilkinson-Berka, J.L. 2006. Angiotensin and diabetic retinopathy. Int. J. Biochem. Cell Biol. 38:752-765.

44. Rattner, A., and Nathans, J. 2006. Macular degeneration: recent advances and therapeutic opportunities. Nat. Rev. Neurosci. 7:860-872.

45. Lund, R.D., et al. 2006. Human embryonic stem cell-derived cells rescue visual function in dystrophic RCS rats. Cloning Stem Cells. 8:189-199.

46. Caplan, A.I., and Dennis, J.E. 2006. Mesenchymal stem cells as trophic mediators. J. Cell. Biochem. 98:1076-1084.

47. Chiaramonte, M.G., Donaldson, D.D., Cheever, A.W., and Wynn, T.A. 1999. An IL-13 inhibitor blocks the development of hepatic fibrosis during a T-helper type 2-dominated inflammatory response. J. Clin. Invest. 104:777-785.

48. Chiaramonte, M.G., et al. 2003. Regulation and function of the interleukin 13 receptor alpha 2 during a $\mathrm{T}$ helper cell type 2-dominant immune response. J. Exp. Med. 197:687-701.

49. Chiaramonte, M.G., Cheever, A.W., Malley, J.D., Donaldson, D.D., and Wynn, T.A. 2001. Studies of murine schistosomiasis reveal interleukin-13 blockade as a treatment for established and progressive liver fibrosis. Hepatology. 34:273-282. 